\title{
First experiments on applying the gasdynamic ECR ion source for negative hydrogen ion production
}

\author{
R.L. Lapin ${ }^{1}$, V.A. Skalyga ${ }^{1}$, I.V. Izotov ${ }^{1}$, S.V. Golubev ${ }^{1}$, S.V. Razin ${ }^{1}$, O. Tarvainen ${ }^{2}$ \\ ${ }^{1}$ Institute of Applied Physics of Russian Academy of Sciences, Nizhny Novgorod, Russia, lapin@ipfran.ru \\ ${ }^{2}$ University of Jyväskylä, Department of Physics, Jyväskylä, Finland
}

\section{Introduction}

$\mathrm{H}^{-}$ion sources are of great demand for charge exchange injection into cyclotrons and storage rings. It has been recently demonstrated that a gasdynamic ECR ion source based on ECR discharge in a simple mirror trap is very efficient for proton beam production [1-2]. Therefore, it was suggested to use the gasdynamic plasma source as the first stage of an $\mathrm{H}^{-}$ion source based on volumetric production through dissociative electron attachment [3-4] (DEA).

The first experiments were performed in a pulsed mode with $37 \mathrm{GHz}$ / up to $100 \mathrm{~kW}$ gyrotron radiation in a dual-trap magnetic system, consisting of two identical simple mirror traps. The schematic of the experimental facility is presented in fig. 1. The first trap was used for plasma production under ECR conditions. Dense hydrogen plasma flux (with estimated current density of about $1-5 \mathrm{~A} / \mathrm{cm}^{2}$ ) from the first trap was allowed to flow into the second trap through a perforated conducting plate. The grid placed between the traps prevents microwave leakage into the second part to avoid electron heating there. Such configuration was chosen to produce a flux of "hot" electrons (energy 50-100 eV), which can effectively ionize and excite high vibrational states of hydrogen molecules in the second trap, and to produce anions there as the result of DEA with "cold" electrons (energy $<10 \mathrm{eV}$ ). Thus, the plasma confined in the second trap presumably consists of two electron populations enabling the volume production of $\mathrm{H}^{-}$ions. Such approach is similar to the one suggested in [5] where heating $2.45 \mathrm{GHz}$ ECR discharge was used as a plasma cathode producing "hot" electrons. A simple two-electrode extraction system was used for the ion beam extraction. Diameters of a plasma electrode and a puller were 5 and $10 \mathrm{~mm}$ respectively. The plasma chamber and plasma electrode had a negative potential up to $-15 \mathrm{kV}$ with respect to the ground. To reflect electrons extracted from the plasma together with the negative ions two pairs of rectangular permanent magnets were installed just after the puller. Both magnet pairs produced a magnetic field transversal to the extracted beam and had opposite directions to compensate each other's influence on the ion trajectories. Coils of the dual-trap magnetic system were able to provide magnetic field maximum ranging from 1 to $2.5 \mathrm{~T}$. Thus it was possible to observe the role of ECR heating in the first trap (ECR field for $37.5 \mathrm{GHz}$ is $1.34 \mathrm{~T}$ ). In the experiments two schemes of neutral gas injection were used: pulsed gas injection into the first trap and constant gas feeding into the first trap through a calibrated leak combined with pulsed gas injection into the second trap. Pulsed gas injection was realized with a controlled valve connected to a buffer vessel with pressure from 0.05 to 1 bar. The second gas-feeding scheme was used to per- form experiment with significantly different neutral gas pressures in each trap as a result of the delay between the valve opening and microwave pulse beingless than the time necessary for the gas molecules to travel to the first trap with sound velocity.

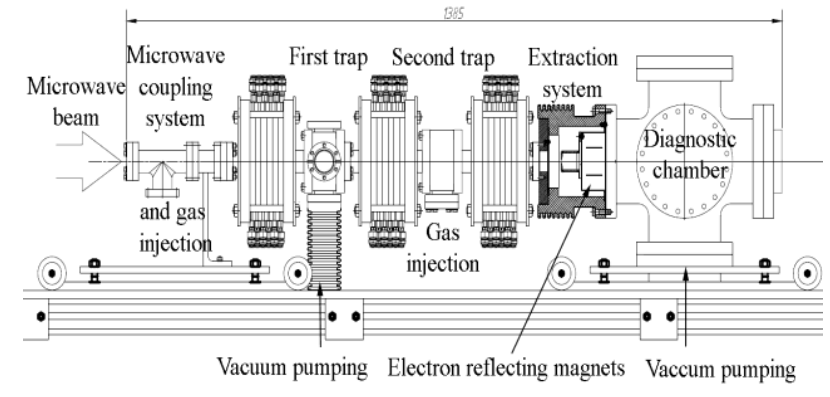

Fig. 1. Scheme of the experimental facility

The main diagnostic tools were a magnetostatic analyzer and a Faraday cup. The analyzer was used for spectrum measurements, determination of impurity level and investigation of $\mathrm{H}^{-}$current dependence on various parameters. Transmission of the analyzer beam line is low and unknown, thus measured currents of certain ions to determine its real current in the extracted beam. The Faraday cup placed right after the extraction system and electron deflection magnets was used for total negative ion current measurements.

Measurements were done in a wide range of numerous parameters, such as gyrotron power, gas injection scheme, magnetic field strength, delay of gas pulse with respect to the microwave radiation pulse.

\section{Experimental results}

As the first step experiments with the pulsed gas injection into the first chamber without any injection into the second one were conducted. In this case the example of a typical ion beam spectrum is presented in fig. 2 . It is clearly seen that a dramatic level of water-induced impurities, detrimental for $\mathrm{H}^{-}$production, were observed. Such problem is connected to the low repetition rate $(0.1 \mathrm{~Hz})$ of the experimental facility and its disposal in future would probably enhance the $\mathrm{H}^{-}$generation efficiency. Meanwhile $\mathrm{OH}^{-}$is considered as the main negative ion and its dependencies on experimental parameters were studied together with $\mathrm{H}^{-}$.

The dependence of $\mathrm{H}^{-}$and $\mathrm{OH}^{-}$currents on microwave heating power is shown in fig. 3. It was shown that, as expected, the $\mathrm{H}^{-}$current depends linearly on the gyrotron power.

The currents of the negative ions depended on magnetic field as shown in fig. 4 . The $\mathrm{H}^{-}$current was significantly higher under the condition of ECR heating, but a 
non-zero signal was observed even at fields below the ECR.

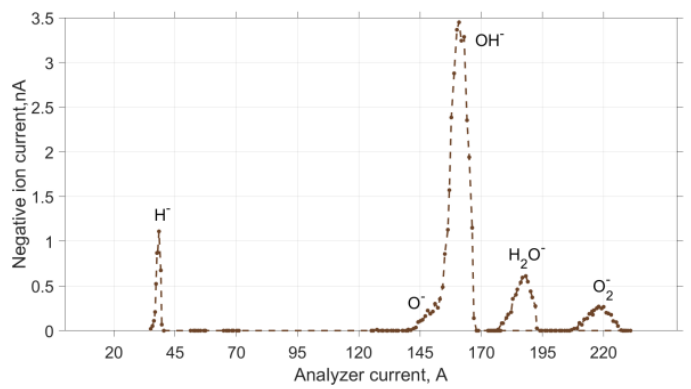

Fig. 2. Negative ion spectrum $(\mathrm{B}=1.6 \mathrm{~T}$, microwave power $80 \mathrm{~kW})$

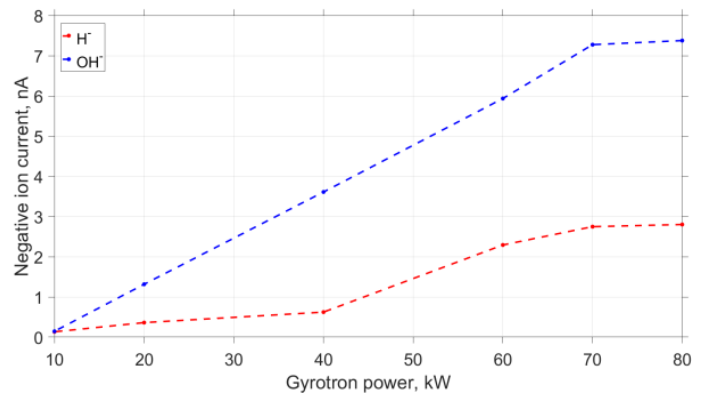

Fig. 3. The dependence of $\mathrm{H}^{-}$and $\mathrm{OH}^{-}$current on gyrotron power $(\mathrm{B}=1.48 \mathrm{~T})$

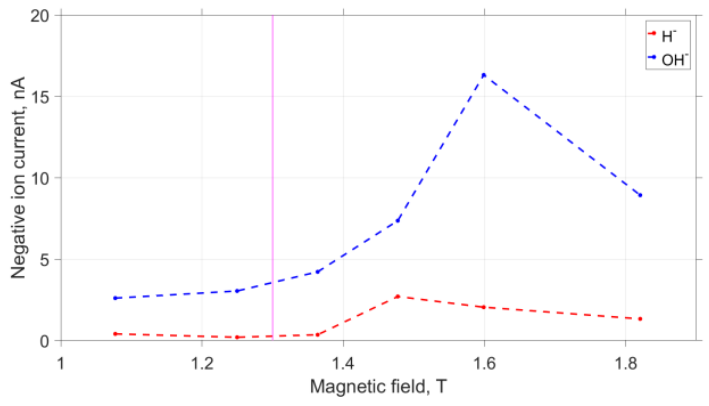

Fig. 4. The dependence of $\mathrm{H}^{-}$and $\mathrm{OH}^{-}$current on magnetic field $\left(\mathrm{P}_{\text {gyrotron }}=80 \mathrm{~kW}\right)$

Data of figures 3 and 4 was collected in case of optimized neutral gas injection. The $\mathrm{H}^{-}$current dependence on microwave pulse delay from the gas valve opening is presented in fig. 5 for different buffer vessel pressures.

Measurements of the total negative ion current with the Faraday cup have shown it at the level of $0.11 \mathrm{~mA}$.

When the second scheme of neutral gas injection was tested, an increase of the total negative ion current by a factor of 15 was observed. In this case the gas pressure in the second trap was significantly increased without affecting the main discharge properties in the first trap. A typical waveform of the current is presented in fig. 6, showing a peak current of approximately $2 \mathrm{~mA}$.

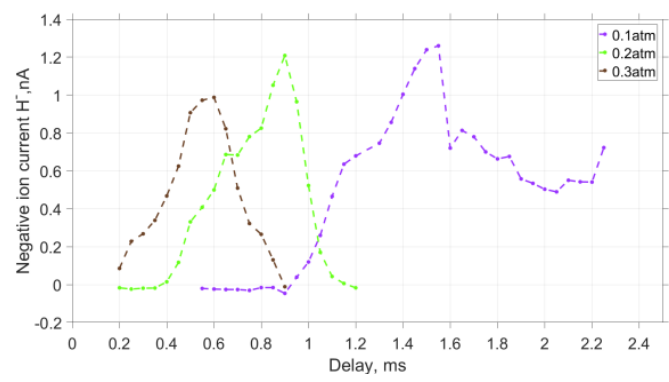

Fig. 5. The dependence of $\mathrm{H}^{-}$current on the delay between the gas valve and microwave radiation pulses for different $\mathrm{H}_{2}$ pressures in the buffer vessel $\left(B=1.6 \mathrm{~T}, \mathrm{P}_{\text {gyrotron }}=80 \mathrm{~kW}\right)$

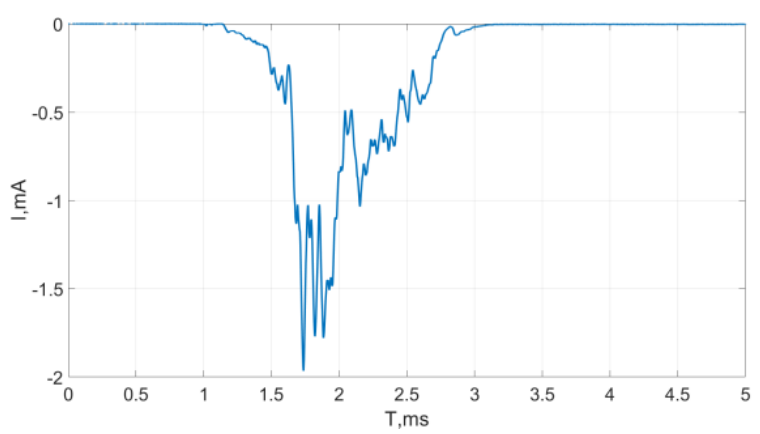

Fig. 6. Typical negative ion current waveform. Microwave power $80 \mathrm{~kW}$, constant gas flow into the first trap and pulsed injection into the second one

Such increase in the total ion current could be connected with higher density of "cold" electron density induced by higher neutral gas pressure. Further experiments would be devoted to more detailed studies of this regime and better optimization of experimental conditions.

Research was carried out with the support of the Federal Agency for Scientific Organizations in the frame of state order No. 0035-2014-0026.

\section{References}

1. Skalyga, V. A., Izotov, I. V., Razin, S.V., Sidorov, A. V., Golubev, S.V., Kalvas, T., Koivisto, H., Tarvainen, O. High current proton beams production at Simple Mirror Ion Source 37 // Rev. Sci. Instrum. 2014. V. 85, No. 2. P. 02A702-1-3.

2. Skalyga, V. A., Izotov, I. V., Golubev, S.V., Sidorov, A. V., Razin, S.V., Vodopyanov, A.V., Tarvainen, O., Koivisto, H., Kalvas, T. New progress of high current gasdynamic ion source // Rev. Sci. Instrum. 2016. V. 87. P. 02A716-1-4.

3. Bacal, M., Hamilton, G. W. H- and D- production in plasmas // Phys. Rev. Lett. 1979. V. 42, No. 1538.

4. Bacal, M. Physics basis and future trends for negative ion sources / M. Bacal // Nuclear Fusion. 2008. Vol. 79, N. 2.

5. Keller,R., Luft, P., Regis, M., Wallig, J., Monroy, M., Ratti, A., Syversrud, D., Welton, R., Anderson, D. A Hybrid IonSource Concept for a Proton Driver Front-End // AIP Conf. Proceedings 773, American Inst. Of Physics, Melville, NY. 2005. P. 104-106. 